\title{
Recent Fusion Research in the National Institute for Fusion Science ${ }^{*}$
}

\author{
Akio KOMORI, Satoru SAKAKIBARA, Akio SAGARA, Ritoku HORIUCHI, \\ Hiroshi YAMADA, Yasuhiko TAKEIRI and NIFS Team \\ National Institute for Fusion Science, Toki 509-5292, Japan
}

(Received 13 July 2011 / Accepted 19 October 2011)

\begin{abstract}
The National Institute for Fusion Science (NIFS), which was established in 1989, promotes academic approaches toward the exploration of fusion science for steady-state helical reactor and realizes the establishment of a comprehensive understanding of toroidal plasmas as an inter-university research organization and a key center of worldwide fusion research. The Large Helical Device (LHD) Project, the Numerical Simulation Science Project, and the Fusion Engineering Project are organized for early realization of net current free fusion reactor, and their recent activities are described in this paper. The LHD has been producing high-performance plasmas comparable to those of large tokamaks, and several new findings with regard to plasma physics have been obtained. The numerical simulation science project contributes understanding and systemization of the physical mechanisms of plasma confinement in fusion plasmas and explores complexity science of a plasma for realization of the numerical test reactor. In the fusion engineering project, the design of the helical fusion reactor has progressed based on the development of superconducting coils, the blanket, fusion materials and tritium handling.
\end{abstract}

(C) 2011 The Japan Society of Plasma Science and Nuclear Fusion Research

Keywords: NIFS, LHD, heliotron, simulation, fusion engineering, helical reactor

DOI: $10.1585 /$ pfr.6.2102149

\section{Introduction}

The National Institute for Fusion Science (NIFS) was established on May 29, 1989 aimed at academic approaches towards the exploration of fusion science for a steady-state helical reactor and establishment of a comprehensive understanding of toroidal plasmas as an interuniversity research organization and a key center of worldwide fusion research. Especially, projects of the Large Helical Device (LHD), simulation science and fusion engineering and design mainly have been pursued to promote scientific understanding, technology, human resource development and education through collaborations with universities and institutions. The above three projects are organized for early realization of the helical fusion reactor, and their activities contribute to the design work.

The recent activities of each project are mainly introduced in this paper. The recent progress of LHD experiments is briefly described in Section 2. In Section 3, the activity of the Numerical Simulation Science Project is shown, and the developments of fusion engineering are described in Section 4. A discussion and a summary are given in the final section.

\section{Large Helical Device Project}

The LHD is a heliotron-type device employing large-

author's e-mail: komori@lhd.nifs.ac.jp

*) This article is based on the presentation at the 20th International Toki Conference (ITC20). scale superconducting magnets to enable advanced studies of net-current-free plasmas [1,2]. The major goal of the LHD experiment is to demonstrate the high performance of helical plasmas in a reactor relevant plasma regime for verifying the potential capability of a fusion reactor. The following missions are declared [3]:

(1) realization of plasmas with high fusion triple product and execution of extensive confinement studies

(2) demonstration of a stable long-pulse discharge

(3) clarification of the role of the radial electric field in plasmas with high ion temperature

(4) realization of beta as high as 5\% and exploration of related physics

(5) control of edge plasmas by divertor for a stable long pulse discharge

(6) investigation of high energy particles simulating alpha particles

The original target plasma parameters are set at $T_{\mathrm{i}}>$ $10 \mathrm{keV}$, averaged beta $\langle\beta\rangle>5 \%$, fusion triple product $n_{\mathrm{e}} \tau_{\mathrm{E}} T_{\mathrm{i}}>10^{20} \mathrm{keV} \cdot \mathrm{m}^{-3} \cdot \mathrm{s}$ and a steady state operation of more than 1 hour (3 MW).

Since 1998, the LHD has been producing highperformance plasmas comparable to those of large tokamaks, based on the reliability of cryogenic systems [4] and the technology development of heating devices. In the past 12 years, the superconducting coils have been excited 1,380 times without any serious problem and the confining magnetic field has been provided with up to 

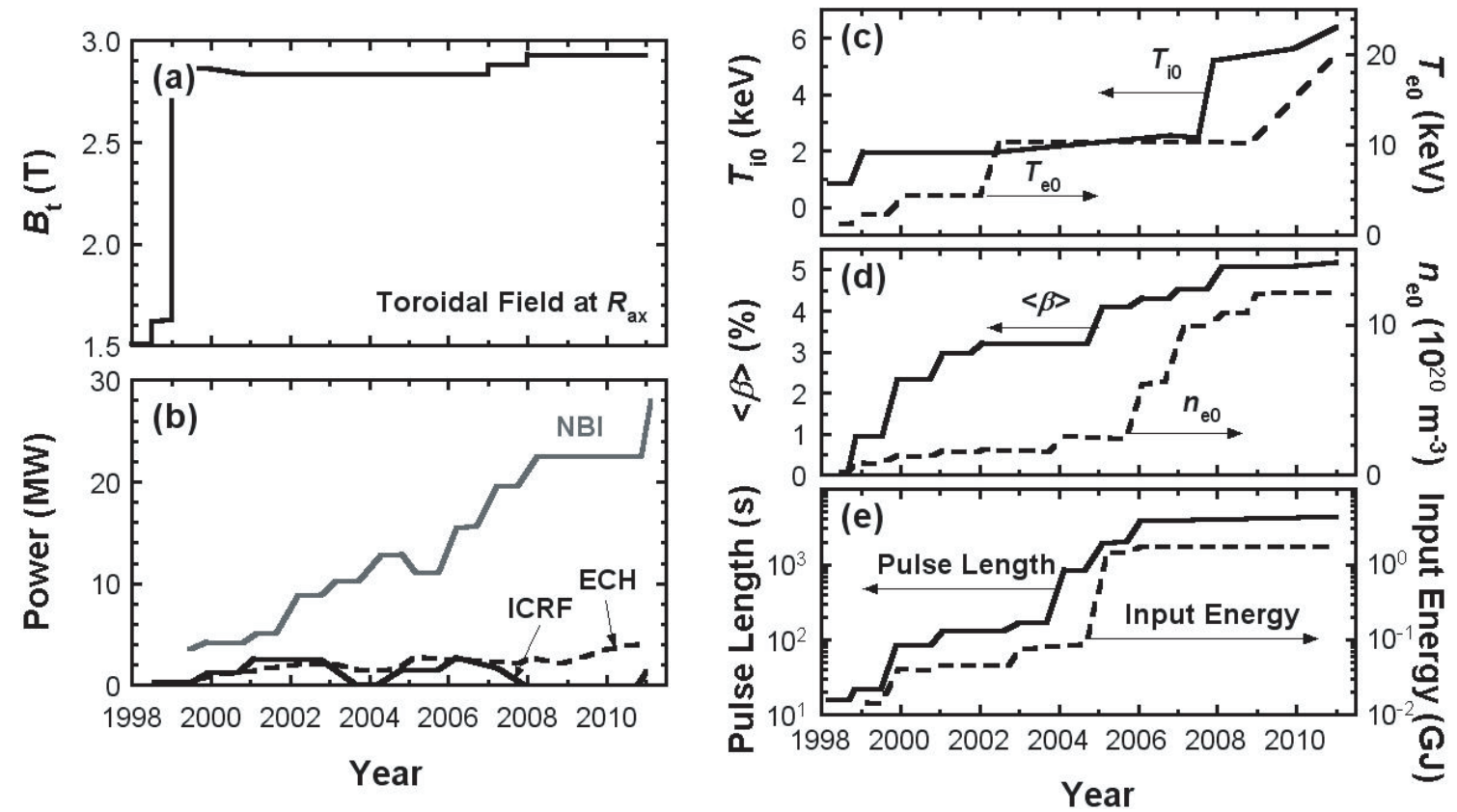

Fig. 1 The development of (a) available troidal field, (b) powers of NBI, ECH and ICRF, (c) central ion and electron temperatures, (d) achieved volume averaged beta and central electron density, (e) pulse length of discharges and input energy of LHD in 1998-2011.

$2.96 \mathrm{~T}$ in steady-state as shown in Fig. 1 (a), which realized 101,000 plasma discharges [5]. Three kinds of heating devices are equipped with LHD, that is, neutral beam injection (NBI) [6], electron cyclotron heating (ECH) [7] and Ion cyclotron heating frequency (ICRF) [8]. Figure 1(b) shows the development of the powers of NBI, ECH and ICRF. Three tangential beams with negative ion sources with the acceleration voltage of $180 \mathrm{keV}$ generate $16 \mathrm{MW}$ in total. The two additional perpendicular beams with positive ion sources of $40 \mathrm{keV}$ were added in 2008 and 2010, for the direct ion heating. The total heating power of NBI has reached about $27 \mathrm{MW}$. The ICRF system greatly contributes to produce the steady-state operation. The power of ICRF itself achieves $1.6 \mathrm{MW}$ for $5000 \mathrm{~s}$. Recently, a high power gyrotron of the ECH system has been successfully developed in collaboration with Tsukuba university. The output power of $1.8 \mathrm{MW}$ was steadily obtained for one second in a $77 \mathrm{GHz}$-gyrotron (Fig. 2).

Figure $1(\mathrm{c}-\mathrm{e})$ show the development of the plasma parameters in 1998-2011. Central $T_{\mathrm{i}}$ achieved $6.4 \mathrm{keV}$ at $1.6 \times 10^{19} \mathrm{~m}^{-3}$ by applying the perpendicular NBI in 2011. Central $T_{\mathrm{e}}$ increased after the finding of an electron internal transport barrier (ITB) $[9,10]$ and approached about $20 \mathrm{keV}$ with the high-power gyrotron. The $\langle\beta\rangle$ increases with the heating power of NBI year by year and has reached $5.1 \%$ [11]. The operation with multi-pellet injection can produce the plasma with peaked density profile with an internal diffusion barrier (IDB) $[12,13]$. The high central electron density of $1.2 \times 10^{21} \mathrm{~m}^{-3}$ has been achieved in an IDB plasma. The pulse length of the discharge has been extended by ECH and ICRF. The input heating energy approached $1.6 \mathrm{GJ}$, which is a world record.

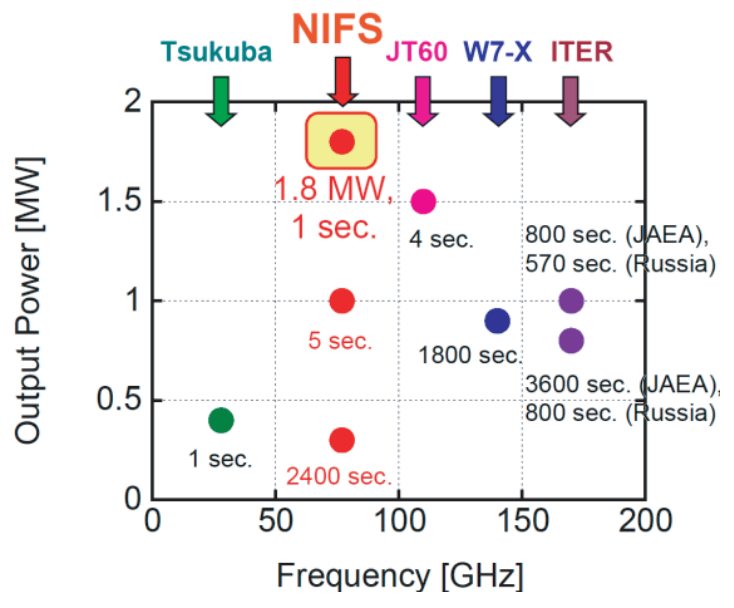

Fig. 2 Development of high power gyrotron.

\subsection{Physics studies to attain the objectives}

The physics studies for attaining these objectives have been greatly developed over the last 12 years. Global energy confinement in the magnetic configuration optimized to neoclassical theory has been proven to be comparable to those of tokamaks in ELMy H-mode, which shows a gyro-Bohm-like property as seen in international stellarator scaling [14]. No significant collisionality dependence as predicted by neoclassical theory has been observed [15]. The geometrical optimization has also demonstrated that anomalous transport is reduced simultaneously.

The formation of a radial electric field is expected to improve the transport properties. The experiments indicate that the large positive electric field ("electron root") by on axis ECH forms the internal transport barrier. The tem- 


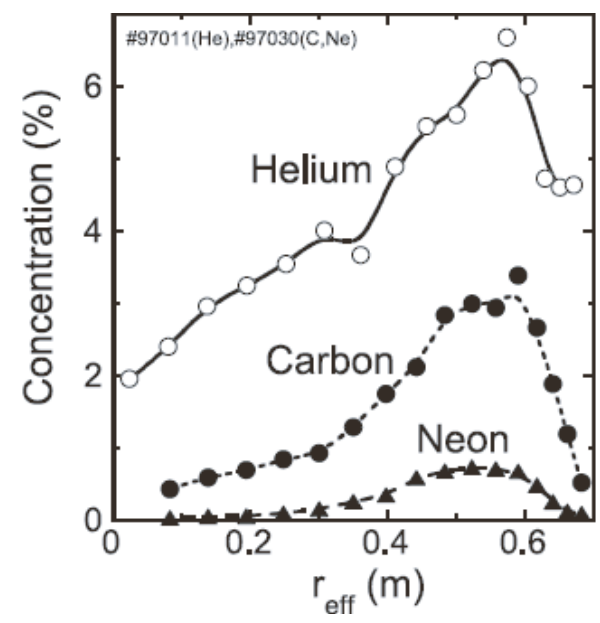

Fig. 3 Radial profiles of Helium, Cabon and Neon densities normalized to electron density.

perature linearly increases with the power, which means no power degradation in the electron root plasmas. The confinement of high energy ions is one of the key issues in helical plasmas because of the existence of helical ripples, and it has been verified by ICRF heated plasma. The high energy ion tail was observed in the energy range up to $2.5 \mathrm{MeV}$, which was measured with a Neutral Particle Analyser (NPA) in steady state operation [16]. Also full orbit Monte-Carlo calculations show that high energy particles of $\mathrm{MeV}$ order are well confined in LHD.

Systematic improvement of RF facilities has enabled stable injection of heating power in steady state. The plasma with $T_{\mathrm{e}}$ and $T_{\mathrm{i}}$ of about $1 \mathrm{keV}$ was successfully maintained by 1 hour by ICRF of $400 \mathrm{~kW}$ and ECH of $100 \mathrm{~kW}[17]$.

\subsection{New findings}

In LHD experiments, three desirable new findings for realization of a helical reactor have been obtained. The first one is the realization of extremely hollow impurity profiles. The profiles have been observed in the steep gradient of ion temperature, which is called "impurity hole" [18]. Figure 3 shows the relative concentration of Helium, Carbon and Neon gases with respect to electron density. The profiles of these gases have an "impurity hole". Experiments show that outward convection is increased with increasing ion temperature gradient in spite of the negative radial electric field, which is opposite the tendency predicted by neoclassical theory. Therefore, the impurity hole is supposed to be driven by anomalous convection [19].

The second is related to the beta limit due to stability. The heliotron configuration has a magnetic hill in the periphery, which destabilizes resistive interchange modes. Linear theory suggest that an increment of beta increases the growth rate of the mode further. However, in the beta range of up to 5\%, no significant MHD activity which deteriorates plasma confinement has been found [20]. MHD

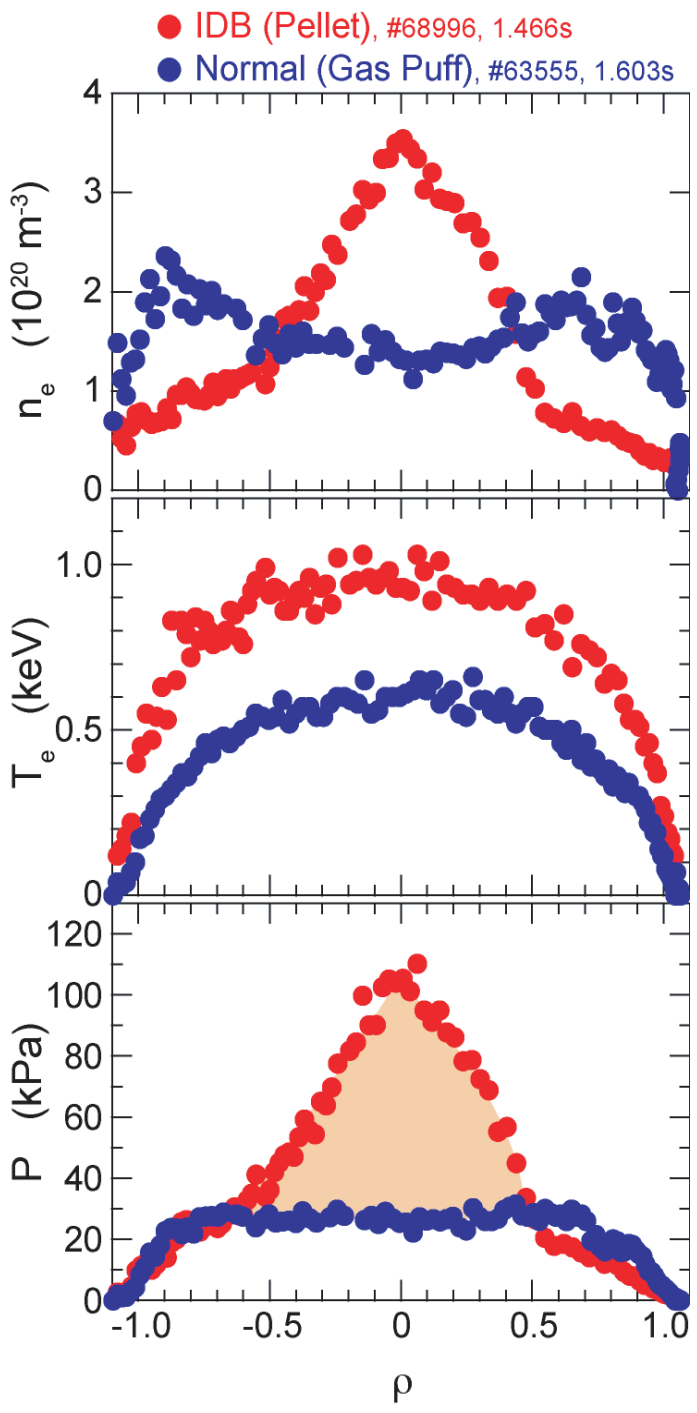

Fig. 4 Comparisons of ne, Te P profiles between the gas-puff and multi pellet cases.

modes are suppressed from the inner to the outer region with increasing beta [21], and the amplitude of the mode clearly depends on magnetic Reynolds number, $S$. This tendency means further stabilization of the mode in high$S$ regime, which corresponds to the reactor regime. The high-beta state is successfully maintained for more than $100 \tau_{\mathrm{E}}$ without strong MHD activity.

The final one is related to discovery of a new operational mode. A peaked high density profile with IDB has been realized by multi-pellet injections. The density is much higher than the usual gas-fueled plasmas. The differences of $n_{\mathrm{e}}, T_{\mathrm{e}}$ and $P$ profiles in both cases are shown in Fig. 4. In the case of multi-pellet injection, the central electron density is much higher than the gas-puff case and the diffusion barrier is formed around $\rho$ of 0.5 although the profile shape of $T_{\mathrm{e}}$ is almost similar to the gas-puff case. The central density of $1.2 \times 10^{21} \mathrm{~m}^{-3}$ at $2.5 \mathrm{~T}$, and central beta of $10 \%$ have been achieved. This high-density plasma has been sustained over $3 \mathrm{~s}$, and the suppression of the im- 


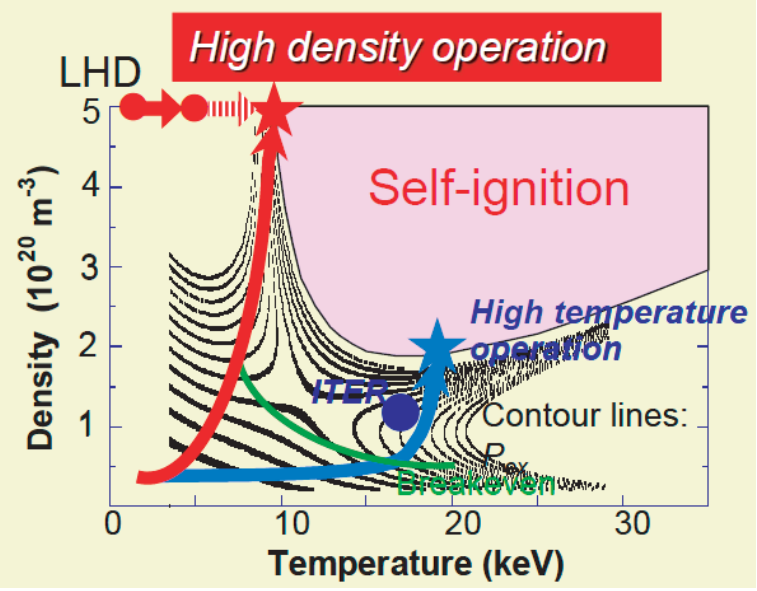

Fig. 5 High density operation on the density and temperature diagram.

purity contamination in the plasma edge with the stochastic region has been found, which is predicted to be due to impurity screening due to friction force in the stochastic layer.

This super high density core (SDC) operation enable us to consideration of new reactor scenario. Figure 5 shows the approach to self-ignition by using SDC operation. This approach is different from the conventional hightemperature scenario, and the plasma with the temperature of $7 \mathrm{keV}$ and density of $6 \times 10^{21} \mathrm{~m}^{-3}$ is targeted. This is expected to mitigate the access to the self-ignition regime because the required temperature is quite a bit lower than the conventional scenario.

\subsection{Future plan}

In order to extend the operational regime and achieve the target parameters further, we have the following plans in the near future:

(1) Steady State Operation with higher heating power

(2) Deuterium-deuterium (DD) Experiments

(3) Effect of Closed Divertor System on Plasma Confinement

The steady state operation by ICRF with $3 \mathrm{MW}$ for more than 1 hour is planned. The DD experiments are planed so as to realize the confinement improvement and to realize higher ion temperature plasmas. The installation of a full closed divertor system is planned for impurity control, stable divertor detachment and so on [22]. $80 \%$ of the inboard divertor area will be modified to the closed configuration with in-vessel pumping by 2012-2013. Figure 6 shows the schematic view of the structure of the closed divertor system. The cryosorption pump system with a pumping speed of $100 \mathrm{~m}^{3} / \mathrm{s}$ and with an $\mathrm{LN}_{2}$ cooled chevron baffle will be applied. In 2011, the partially closed divertor was installed to demonstrate the neutral compression. The neutral pressure in the closed divertor reached

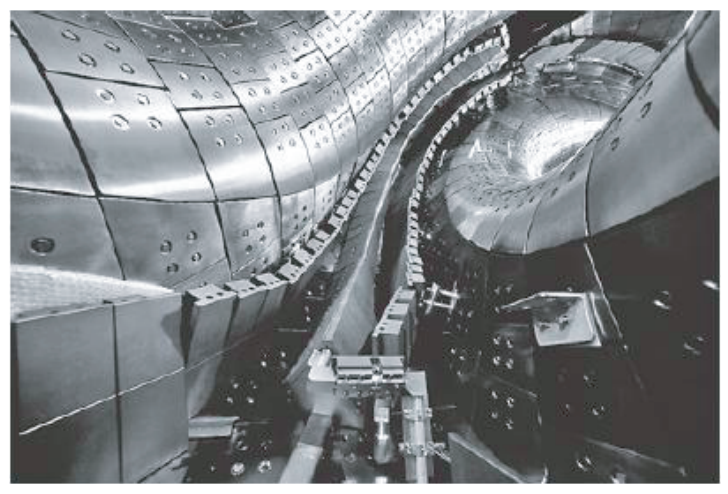

Fig. 6 Structure of closed divertor system.
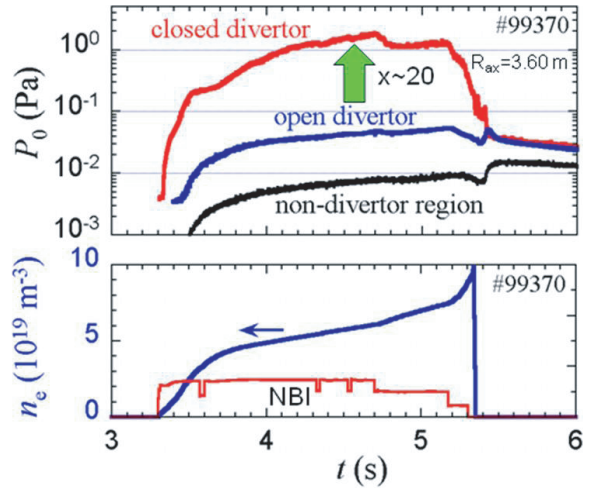

Fig. 7 Difference of neutral pressures in closed, open divertor and non-divertor region.

more than $1 \mathrm{~Pa}$, which is about 20 times higher than that in open divertor region and 100 times higher than that in nondivertor region as shown in Fig. 7. The maximum compression was obtained in a neoclassically optimized configuration with an inward shifted magnetic axis.

In addition to the above subjects, research for physics understanding will be pursued to obtain further knowledge of three-dimensional currentless plasma physics.

\section{Simulation Science Project}

The simulation science project has been established so as to understand and systemize the physical mechanisms of plasma confinement in fusion plasmas including magnetic confinement and laser systems and to explore the complexity science of a plasma as a nonlinear, non-equilibrium, open system. The final goal of this project is to predict the behaviour of plasmas in toroidal magnetic confinement devices on all relevant scales both in time and space for realization of an LHD numerical test reactor (LHD-NTR) $[23,24]$. The concept of LHD-NTR aims to create a fusion reactor in virtual reality in order to optimize physics, engineering and economy. In order to realize this concept, we need an integrated modeling of multi-scale physics, which is called a hierarchy-renormalized model, based on the understanding of complexity physics as shown in 


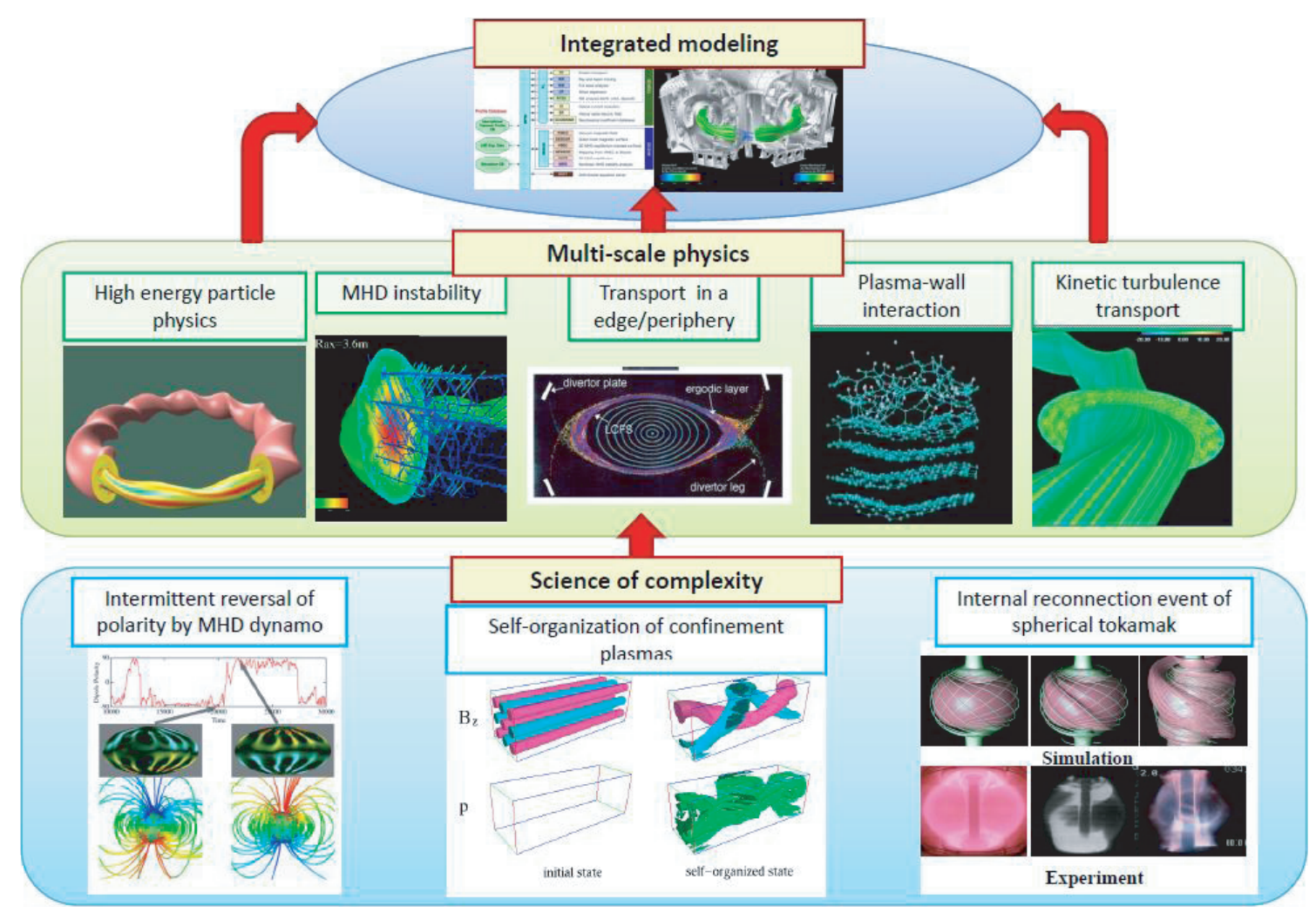

Fig. 8 Evolution of simulation science.

Fig. 8. The hierarchy-renormalized simulation model consists of the hierarchy-integrated simulation model and the hierarchy-extended simulation model. The former model is based on the transport model, which is suitable to interpret the experimental observation of macro physics quantities. The hierarchy-extended simulation model is to understand cross-hierarchy interactions (see Ref [23] in detail).

For developing the LHD-NTR, many simulation works have been successfully advanced in order to understand multi-physics in fusion plasmas and complexity science, as shown Fig. 8. We need all elements of the physics controlling fusion plasmas and innovative numerical technologies to interlock them, together with powerful supercomputing resources at the Petascale level or more as a common platform of simulation science. And we should assemble all obtained results to upgrade integral transport models and approach the final NTR in the synergy of experimental groups and fusion engineering groups.

Several works are briefly described as follows: The gyro-kinetic Vlasov simulations of ion temperature gradient (ITG) turbulence and zonal flows have been done in the LHD configuration [25]. The simulation results indicate that the given zonal flows have higher levels for longer time in the inward-shifted configuration of the magnetic axis than that in the outward shifted one, which is consistent with the experimental observation $[26,27]$. The simulation code for investigating an interaction between MHD and energetic particles, MEGA, was extended with the ion Larmar radius effect, and was applied to calculate the lin- ear growth rate and the damping rate of the $n=4$ toroidal Alfvén eigenmode (TAE) in the TFTR D-T plasma [28]. The obtained results are consistent with the calculation of the NOVA-K code [29]. Recently, the extended MEGA code has been applied to investigate the non-linear evolution of TAE in an ITER-like plasma [28]. The non-linear evolution of MHD instabilities has been investigated by numerical simulation of the fully three-dimensional MHD code, MINOS [30]. This simulation has been carried out for linear unstable configuration of LHD, and clarified that the instability leads to the local flattening of the pressure and releases the kinetic energy in the direction parallel to the magnetic field line. The compressibility effect reduces the linear growth rates of the instability.

In addition, multi-scale interactions among the MHD instability, micro turbulence and zonal flow have been investigated based on two fluid equations in tokamak configurations [31,32]. The magnetic reconnection, which is known as a multi scale phenomenon, has been investigated by using a multi-hierarchy simulation model $[33,34]$. The simulation works with regard to pellet injection [35], plasma-wall interaction including blob dynamics [36] and molecular dynamics [37] have been successfully developed. These works are expected to contribute the development of the LHD-NTR.

\section{Fusion Engineering Project}

In the fusion engineering project, four key subjects for 
the early realization of the helical fusion reactor have been researched: developments of superconducting coils, material and blanket, tritium handling, and the design of helical fusion reactor. The investigation of these subjects has been developed in parallel with the design work of helical fusion reactor based on LHD experiments.

The application of superconducting technology to a commercial fusion reactor is absolutely imperative from the viewpoint of the economic efficiency and compactness. The objectives of our researches are (1) improvement of LHD superconducting system, (2) development of a helical reactor system and the elemental technology, (3) basic study for the construction of a superconducting system and (4) the application research of fusion technology to other fields.

As described at Section 2, the high reliability of the LHD superconducting system itself has been demonstrated through actual experiments for the past 12 years. In FY2006, the cryogenic system was reconstructed for the sub-cooling of helical coils, which realizes a higher magnetic field. Both inlet and outlet temperatures were successfully decreased to $3.2 \mathrm{~K}$ and $3.8 \mathrm{~K}$, respectively, and the maximum magnetic field of $2.96 \mathrm{~T}$ became available [5]. This sub-cooling system has been safely running for over 7000 hours. The operational experience and the high reliability contribute the design of the superconducting system for the helical fusion reactor [38].

The system for current control of the superconducting coils was improved for the temporal movement of the magnetic axis during a discharge [39]. The capacity of the power supply of the poloidal coils was increased, which enables motion of $R_{\mathrm{ax}}$ by $5 \mathrm{~cm} / \mathrm{s}$. This system was mainly applied to the experiments for high-beta plasma production and MHD study [11].

In a helical fusion reactor, the construction of the cooling system with a low pressure loss of liquid Helium is a major crucial issue because the superconducting magnets with the major radius of more than $14 \mathrm{~m}$ and the maximum magnetic field of about $13 \mathrm{~T}$ are required in the present plan. In order to resolve this problem, the indirectly-cooled superconductor has been proposed and developed [40]. The planned superconductor has a square cross section with $50 \mathrm{~mm} \times 50 \mathrm{~mm}$ and the maximum current is about $100 \mathrm{kA}$. The superconducting $\left(\mathrm{Nb}_{3} \mathrm{Al}\right)$ and copper wires are bundled and covered by an Aluminumalloy jacket. The jacket is agglutinated by the Friction Stir Welding method. For demonstrating the validity of this method, the sample with $17 \mathrm{~mm} \times 5 \mathrm{~mm}$ was fabricated and tested under the conditions with $12 \mathrm{~T}$ and $4.7 \mathrm{kA}$. As a result, no degradation from the critical current was observed. On the other hand, the application of the high temperature superconducting (HTS) magnet is also considered instead to $\mathrm{Nb}_{3} \mathrm{Al}$. The R\&D test of Bi-2223 wire under the conditions with $8 \mathrm{~T}$ and $20 \mathrm{~K}$ indicates the critical current of $10 \mathrm{kA}$. Also it was shown that the HTS conductor has significantly higher stability against heating than the conven- tional superconductor.

The effect of neutron exposure on superconductors is also an important subject for a fusion reactor. This research has been advanced in collaboration with universities and JAEA. For example, changes in the critical current, magnetic field and temperature of several superconducting wires due to $14 \mathrm{MeV}$ neutron exposure with $1.78 \times 10^{21} \mathrm{n} / \mathrm{m}^{2}$ were investigated [41]. A database has been constructed for the modeling. Also, the development of materials with lower radio-activation has been carried out. Especially, superconductors of $\mathrm{V}_{3} \mathrm{Ga}$ and $\mathrm{MgB}_{2}$, having a short half-life, have been successfully fabricated and the validity has been demonstrated in collaboration with universities [42].

The vanadium alloy is expected as one of the attractive materials for fusion materials because of low activation. NIFS has been mainly developing high purity vanadium alloy (V-4Cr-4Ti), NIFS-HEAT, for a long time in collaboration with many universities. The high machinability and weldability of NIFS-HEAT were demonstrated till 2003 [43], whereas the applicability to the blanket has been investigated through various experiments such as neutron exposure. Also the applicability of NIFS-HEAT coated by tungsten to the first wall has been investigated. Tungsten coating has been directly done by the vacuum plasma spray (VPS) method, which is advantageous to coat to structures with complex shape and large area. This test was applied to low activation ferrite steel.

In the blanket, the reduction of the loss of pressure due to the MHD effect is one of the key subjects. In order to increase insulation properties for electricity in liquid lithium, the application of ceramic materials as has been considered [44]. In particular, immersion test of sintered ceramic samples to liquid lithium indicates that $\mathrm{Er}_{2} \mathrm{O}_{3}$ is one of the probable materials. Also it has been experimentally found that the $\mathrm{Er}_{2} \mathrm{O}_{3}$ coating has low corrosion and high insulation characteristics [45].

In the D-T fusion blanket, (1) conversion of high neutron energy to heat, (2) breeding of tritium and (3) shielding of radiation to superconducting coils are required. In order to satisfy these subjects, four kinds of concepts of the blanket, (a) Flibe $\left(\mathrm{LiF}-\mathrm{BeF}_{2}\right)+\mathrm{Be}$, (b) Flibe+cooled STB (Spectral-shifter and Tritium breeder Blanket), (c) Li/Valloy and (d) Flibe/V-alloy have been considered. In addition to investigations into the characteristics of the above blanket materials, transport of neutrons and gamma rays has been calculated by using MCNP code for estimating the effect on materials [46]. The radioactivation characteristics of the blanket materials have been investigated by the neutron exposure.

Based on the above engineering developments, the design of the Force Free Helical Reactor (FFHR) has progressed as an attractive steady state helical fusion reactor [47]. The design of the magnetic configuration is mainly based on the confinement scaling of LHD experiments, and the recent design considering engineering constraints such 


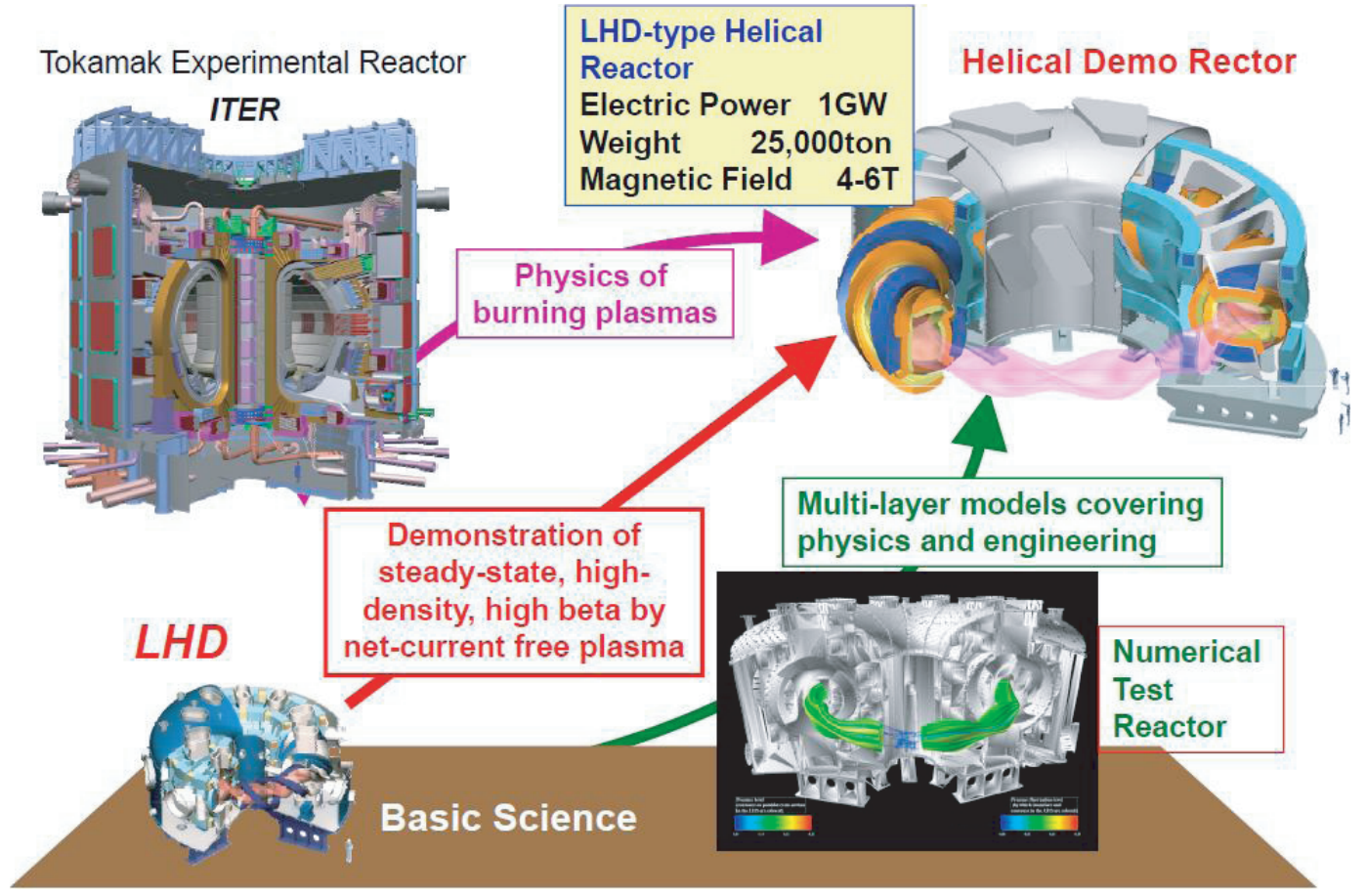

Fig. 9 Plan toward helical demo reactor.

as neutron wall loading shows the optimum major radius and central toroidal field are 14-17 $\mathrm{m}$ and 4-6 T, respectively. The neutron wall loading is predicted to be lower than $2 \mathrm{MW} / \mathrm{m}^{2}$, and the blanket lifetime is about 10 years. If the STB is applied for the first wall, the replacement-free blanket concept is possible. The new ignition scenario, SDC plasma with high-density and low-temperature, has been taken into account as well as the standard scenario with low-density and high-temperature as described at Section 2.2. The operation using the proportional-integrationderivative (PID) feedback control system is proposed in order to avoid thermally unstable operation [48].

\section{Discussion and Summary}

The role of NIFS is to systemize the science related to the helical demonstration power plant (helical DEMO) by steady-state magnetic confinement in collaboration with universities and institutes as well as international collaboration. The understanding of two major issues, burning and steady-state, are required for early realization of a helical DEMO. Therefore, the complementary development of helical devices and the ITER are absolutely imperative. The ITER is expected to provide the knowledge regarding demonstration of ignition and a control of burning plasma, whereas the understanding of steady-state plasma would be obtained in experiments of LHD, W7-X [49] and JT60SA [50]. Also, the numerical test reactor would contribute to the concrete prediction of plasma physics and the engineering (Fig. 9).

Since 1989, NIFS is making an effort to obtain the knowledge of science and technology aiming at the real- ization of a helical fusion reactor through Large Helical Device experiments and theoretical simulation, as well as human resource development.

Large Helical Device experiments have verified the intrinsic ability of Stellarator/Heliotrons with currentless plasmas, and produced new findings contributing to the understanding of common physics in magnetic confinement systems.

The detailed plan aiming at the realization of a helical fusion reactor has been established through physics understanding based on contributions from LHD experiments, simulation science and development of fusion engineering.

\section{Acknowledgments}

The author is grateful to all NIFS staff for their excellent work. He also acknowledges all contributions from international and domestic collaborators.

[1] A. Iiyoshi et al., Nucl. Fusion 39, 1245 (1999).

[2] O. Motojima et al., Nucl. Fusion 40, 599 (2000).

[3] A. Komori et al., Fusion Sci. Technol. 58, 1 (2010).

[4] T. Mito et al., Fusion Eng. Des. 81, 2389 (2006).

[5] S. Imagawa et al., Nucl. Fusion 47, 353 (2007).

[6] Y. Takeiri et al., Fusion Sci. Technol. 58, 482 (2010).

[7] T. Shimozuma et al., Fusion Sci. Technol. 58, 530 (2010).

[8] T. Mutoh et al., Nucl. Fusion 43, 738 (2003).

[9] M. Yokoyama et al., Nucl. Fusion 47, 1213 (2007).

[10] T. Shimozuma et al., Fusion Sci. Technol. 58, 38 (2010).

[11] S. Sakakibara et al., Proc. of 23rd IAEA Fusion Energy Conference (11-16 Oct. 2010, Daejon, Korea) EXS/P5-13.

[12] R. Sakamoto et al., Fusion Sci. Technol. 58, 53 (2010).

[13] N. Ohyabu et al., Phys. Rev. Lett. 97, 05502 (2006). 
[14] H. Yamada et al., Fusion Sci. Technol. 58, 12 (2010).

[15] H. Yamada et al., Plasma Phys. Control. Fusion 43, A55 (2001).

[16] K. Saito et al., Fusion Sci. Technol. 58, 515 (2010).

[17] R. Kumazawa et al., Fusion Sci. Technol. 58, 524 (2010).

[18] K. Ida et al., Nucl. Fusion 49, 095024 (2009).

[19] M. Yoshinuma et al., Proc. of 23rd IAEA Fusion Energy Conference (11-16 Oct. 2010, Daejon, Korea) EXC/9-1.

[20] S. Sakakibara et al., Plasma Phys. Control. Fusion 50, 124014 (2008).

[21] A. Komori et al., Phys. Plasmas 12, 056122 (2005).

[22] S. Masuzaki et al., Plasma Fusion Res. 6, 1202007 (2011).

[23] S. Sudo, Plasma Phys. Control. Fusion 133, 012025 (2008).

[24] N. Nakajima, 2008 lecture at the 2nd Simulation Science Symposium, Tajimi.

[25] S. Ferrando-Margalet, H. Sugama and T.-H. Watanabe, Phys. Plasmas 14, 122505 (2007).

[26] H. Sugama and T.-H. Watanabe, Phys. Rev. Lett. 94, 115001 (2005).

[27] H. Sugama and T.-H. Watanabe, Phys. Plasmas 13, 012501 (2006).

[28] Y. Todo et al., J. Plasma Phys. 72, 817 (2006).

[29] G.Y. Fu et al., Phys. Plasmas 5, 4284 (1998).

[30] H. Miura, N. Nakajima, T. Hayashi and M. Okamoto, Fusion Sci. Technol. 51, 8 (2007).

[31] A. Ishizawa and N. Nakajima, Phys. Plasmas 14, 040702 (2007).
[32] A. Ishizawa and N. Nakajima, Nucl. Fusion 47, 1540 (2007).

[33] R. Horiuchi, H. Ohtani and A. Ishizawa, J. Plasma Phys. 72, 953 (2006)

[34] S. Usami, H. Ohtani and R. Horiuchi, Comm. Comp. Phys. 4, 537 (2008).

[35] R. Ishizaki, P.B. Parks, N. Nakajima and M. Okamoto, Phys. Plasmas 8, 4064 (2006).

[36] S. Ishiguro and H. Hasegawa, J. Plasma Phys. 72, 1233 (2006).

[37] A. Ito, H. Nakamura and A. Takayama, to be published in J. Phys. Soc. Jpn.

[38] S. Imagawa et al., Nucl. Fusion 49, 075017 (2009).

[39] H. Chikaraishi et al., Fusion Eng. Des. 83, 260 (2008).

[40] K. Takahata et al., Fusion Eng. Des. 82, 1487 (2007).

[41] A. Nishimura et al., Fusion Eng. Des. 84, 1425 (2009).

[42] Y. Hishinuma et al., IEEE Trans. Appl. Supercond. 19, 2670 (2009).

[43] T. Nagasaka et al., Fusion Sci. Technol. 44, 465 (2003).

[44] T. Muroga and B.A. Pint, Fusion Eng. Des. 85, 1301 (2010).

[45] M. Kondo et al., Fusion Eng. Des, in press (2011).

[46] T. Tanaka et al., Nucl. Fusion 48, 035005 (2008).

[47] A. Sagara et al., Fusion Eng. Des. 83, 1690 (2008).

[48] O. Mitarai et al., Fusion Sci. Technol. 56, 1495 (2009).

[49] H. Renner et al., Nucl. Fusion 40, 1083 (2000).

[50] Y. Kamada et al., Nucl. Fusion 51, 073011 (2010). 